\section{Enhancing everyday practice}

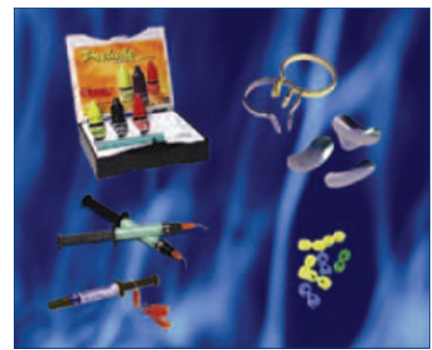

Evident has recently launched a collection of products for minimal adhesive dentistry from American company Danville. All of the products in the range are designed to work with any composite system. The consumable products include Prelude versatile three in one adhesive. This is a self-etch and total-etch system needed for dentine and enamel bonding and usable with light cure, dual cure and self cure composites: Accolade thixotropic flowable composite - which has polishability and good handling qualities - and StarFlow, a highly flowable composite.

Also included is Contact Matrix, a sectional matrix system that makes tight contacts and natural contours easy to achieve, and Contact Wedge, a unique elastic wedge for superior cervical adaptation.

All of Evident's consumable products have been introduced to aid dentists who wish to practise less invasive techniques, both to the benefit of their patients and as an enhancement to everyday working practices. For more information about the new range of consumable products FreeCall Evident on 0500321111.

\section{Total patient contact solution}

A new total patient call system has been launched by healthcare messaging solutions company iPlato, in partnership with Biomni Voice, a provider of automated telephone voice messaging and reminder services.

The new integrated solution means that patients can be automatically contacted by either landline or mobile phone to significantly reduce Did Not Attend (DNA) rates for dental appointments.

Biomni Voice messaging service works by allowing appointment reminder messages to be created and converted to natural sounding speech or by selecting a pre-recorded mes-

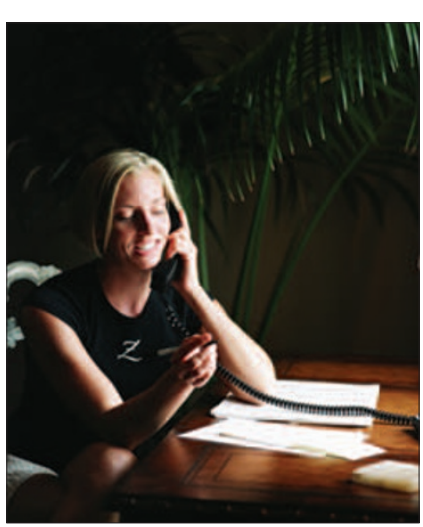
sage using real voice.

The voice service prompts the call recipient to respond immediately viatheirtelephone keypad. All calls and responses are logged and reported to enable appropriate action to be taken when needed. The service will also retry at pre-determined intervals and detect answer machines or voicemail. Visit www.iplato.net for further information.
Suck to stimalate saliva

Molar Ltd has announced the arrival of new bioxtra Sucking Tablets to complement the bioxtra range of products. bioxtra is designed specifically for patients who suffer

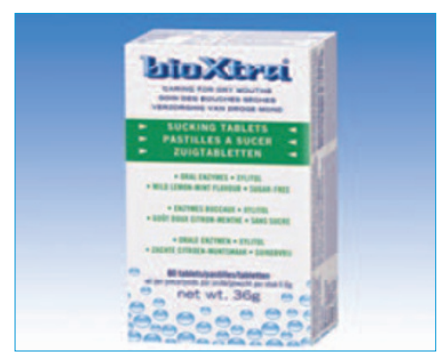
from dry mouth (xerostomia).

This extremely unpleasant condition can result in patients having difficulty speaking, eating and sleeping and can be detrimental to their oral health. The range has been designed to provide patients with a better quality of life, providing comfort, salivary stimulation and hygiene.

The new Sucking Tablets have a mild flavour to give a cooling effect in the mouth and to help stimulate saliva flow. Their active ingredients include xylitol, Lactoperoxidase and Lactoferrin, which are derived from colostrum extract. The recommended price is $£ 4.95$ for a pack of 60 tablets.

Other products include Mild Antibacterial Toothpaste and Alcohol-free Mouth rinse for daily hygiene, Non-stick Dental Gum and the New Sucking Tablets for salivary stimulation and Moisturising Gel and Moisturising Gel Mouth spray (both NHS prescribed) for long-lasting comfort. Patients can buy the whole range from pharmacies or by mail-order from Dental Products 2 U Ltd, telephone 0845226 0660. Samples and a care guide are available on request. Email: info@molarltd.co.uk.

\section{Practical stools}

Stevens Seating Limited offer a range of stools suitable for the surgery. The Model $\mathrm{B}+$ and Model $\mathrm{A}+$ are the latest versions of the Pratique stool.

The Model A+ assistant's stool has an 18 inch aluminium base and is silver finished with an adjustable backrest height. The Model B+ operator's stool has quality dual-wheeled black castors, durable vinyl fabric, gas-lift height adjustment and comes in a wide range of colours. Model C is constructed from quality components and instant left or right-handed gas-lift height adjustment. Visit www.stevens seating.co.uk for more information.

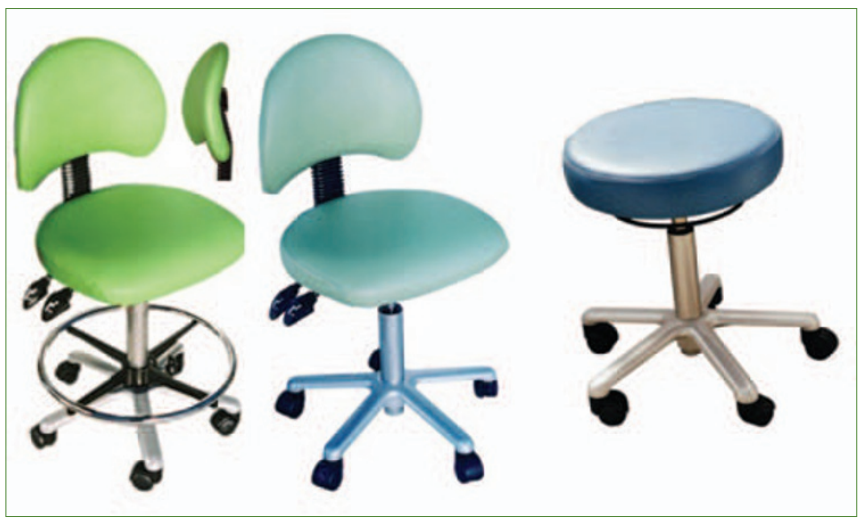

